\title{
Unsteady MHD convective flow within a parallel plate rotating channel with thermal source/sink in a porous medium under slip boundary conditions
}

\author{
G.S. Seth*, R. Nandkeolyar ${ }^{+}$and Md. S. Ansari ${ }^{\#}$ \\ "Department of Applied Mathematics, Indian School of Mines, Dhanbad-826004, INDIA \\ ${ }^{+}$Department of EngineeringMathematics, GITAM University-Hyderabad Campus, Hyderabad-502329, INDIA \\ ${ }^{\#}$ School of Petroleum Technology, Faculty of Mathematics, Pandit Deendayal Petroleum University, Gandhinagar-382007, INDIA \\ "Corresponding Author: e-mail: gsseth_ism@yahoo.com, Tel +91-326-2235421, Fax.+91-326-2296619
}

\begin{abstract}
Unsteady hydromagnetic convective flow of a viscous incompressible electrically conducting heat generating/absorbing fluid within a parallel plate rotating channel in a uniform porous medium under slip boundary conditions is investigated. Exact solution of the governing equations for fully developed flow is obtained in closed form. Expressions for skin friction due to primary and secondary flows and Nusselt number at the plate $\eta=1$ are also derived. Asymptotic behavior of the solution for the fluid velocity is analyzed for large values of frequency parameter $\omega$ to gain some physical insight into the flow pattern. The numerical values of the primary and secondary velocities and fluid temperature are displayed graphically versus channel width variable $\eta$ for various values of pertinent flow parameters whereas numerical values of skin frictions due to primary and secondary flows and Nusselt number at the plate $\eta=1$ are presented in tabular form for different values of pertinent flow parameters.
\end{abstract}

Keywords: Thermal source/sink, slip boundary conditions, periodic pressure gradient, convective flow, magnetic field, rotation, porous medium.

\section{Introduction}

Flow of a viscous fluid in a rotating medium is of considerable importance due to the occurrence of various natural phenomena and for its application in various technological situations which are governed by the action of Coriolis force. The broad subjects of oceanography, meteorology, atmospheric science and limnology all contain some important and essential features of rotating fluids. The viscous fluid flow problems in rotating medium under different conditions and configurations are investigated by many researchers in the past to analyze various aspects of the problem. Mention may be made of the research studies of Greenspan and Howard (1963), Holton (1965), Walin (1969), Siegman (1971), Puri (1974), Puri and Kulshrestha (1974), Mazumder (1991), Ganapathy (1994), Hayat et al (2001), Hayat and Hutter (2004) and Das et al. (2008). The study of simultaneous effects of rotation and magnetic field on the fluid flow problems of a viscous incompressible electrically conducting fluid may find applications in the areas of geophysics, astrophysics and fluid engineering. An order of magnitude analysis shows that, in the basic field equations, the effects of Coriolis force are more significant as compared to that of inertial and viscous forces. Furthermore, it may be noted that Coriolis and magnetohydrodynamic forces are comparable in magnitude and Coriolis force induces secondary flow in the flow-field. Taking into consideration these facts Vidyanidhi (1969), Nanda and Mohanty (1971), Mazumder (1977), Jana et al (1977), Jana and Datta (1980), Prasad Rao et al (1982), Seth and Maiti (1982), Mandal et al (1982), Mandal and Mandal (1983), Raman Rao and Linga Raju (1990), Nagy and Demendy (1993, 1995), Ghosh and Bhattarchjee (2000), Seth and Singh (2008), Seth and Ansari (2009), Seth et al (2009) and Ghosh et al (2009) studied steady MHD flow of a viscous incompressible electrically conducting fluid in a rotating channel under different conditions considering various aspects of the problem. Investigation of oscillatory flow in a rotating channel is important from practical point of view because fluid oscillations may be expected in many MHD devices and natural phenomena where fluid flow is generated due to oscillating pressure gradient or due to 
vibrating walls. Keeping in view this fact Mukherjee and Debnath (1977), Seth and Jana (1980), Singh (2000), Ghosh (1993), Ghosh and Pop (2003), Hayat et al (2004) and Guria et al (2009) investigated oscillatory flow of a viscous incompressible electrically conducting fluid in a rotating channel under different conditions to analyze various aspects of the problem. Rahman and Sattar (1999) studied MHD free convection and mass transfer flow with oscillating plate velocity and constant heat source in a rotating frame of reference. In all these investigations "no-slip" boundary condition is considered for the velocity field. However, in some application e.g. in microfluidic and nanofluidic devices where the surface to volume ratio is large, the slip behavior is more typical and slip boundary condition is usually used for the velocity field (Darhuber and Troian, 2005) which was first proposed by Navier in the year 1823. There exist many physical reasons for the slip over hydrophobic surfaces among which are: molecular slip (Blake, 1990) and small dipole-moment of polar liquids (Melin et al, 2004). Also wall slip can occur in the working fluid which contains concentrated suspensions (Soltani and Tilmazer, 1998). Keeping in view these facts the effects of fluid slippage at the wall for Couette flow under steady state condition for gases are studied by Marques et al (2000) whereas Khaled and Vafai (2004) investigated Stokes and Couette flows produced by an oscillatory motion of a wall under slip boundary conditions. Soundalgekar (1970) considered hydromagnetic fluctuating flow past an infinite porous plate in slip flow regime while Sastry and Bhadram (1976) studied magnetogasdynamic flow past an infinite porous plate in slip flow regime. Makinde and Osalusi (2006) investigated MHD steady flow in a channel with permeable boundaries under slip boundary conditions. Linga Raju (2007) considered steady hydromagnetic flow in a rotating channel with non conducting walls in slip flow regime. Smolentsev (2009) investigated three types of MHD flow problems assuming hydrodynamic slip condition at the interface between the electrically conducting fluid and insulating walls which are: (i) Hartmann flow; (ii) fully developed flow in a rectangular duct and (iii) quasi two dimensional turbulent flow. Abelman et al. (2009a) considered steady MHD flow of a third grade fluid past a rigid plate with slip boundary condition in a rotating frame whereas Abelman et al. (2009b) studied steady MHD Couette flow of thermodynamic compatible third grade fluid filling the porous space in a rotating frame taking partial slip into account.

Unsteady convective flow of a viscous incompressible heat generating/absorbing fluid is of considerable importance due to appreciable temperature difference between the surface and ambient fluid in so many fluid flow problems of physical interest. Internal heat generation/absorption plays significant role in various physical phenomena such as fluids undergoing exothermic or endothermic chemical reactions ( Vajravelu and Nayfeh, 1992), convection in Earth's mantle (McKenzie et al, 1974), application in the field of nuclear energy (Crepeau and Clarksean, 1997), post accident heat removal (Baker et al, 1976), fire and combustion modeling (Delichatsios, 1988) and the development of metal waste from spent nuclear fuel (Westphal, 1994). Although exact modeling of internal heat generation or absorption is quite difficult, some simple mathematical models yet idealized can express its average behavior for most physical situations. Sparrow and Cess (1961) considered temperature-dependent heat absorption in their investigation of steady stagnation point flow and heat transfer. Moalem (1976) studied steady state heat transfer in a porous medium with temperature-dependent heat generation. Jha and Ajibade (2009) considered free convection flow of heat generating/absorbing fluid between vertical porous channel due to periodic heating of the walls of the channel and temperaturedependent heat generation/absorption. Kamel (2001) investigated unsteady MHD convection flow through a porous medium bounded by an infinite vertical porous plate with temperature-dependent thermal source/sink. Chamkha (2004) considered unsteady two dimensional convective heat and mass transfer boundary layer flow of a viscous, incompressible, electrically conducting and heat absorbing fluid past a semi-infinite vertical permeable plate with temperature-dependent heat absorption.

The aim of the present paper is to study unsteady hydromagnetic convective flow of a viscous, incompressible, electrically conducting and heat generating/absorbing fluid within a parallel plate vertical channel in a uniform porous medium under hydrodynamic slip boundary conditions with temperature dependent thermal source/sink when both the fluid and channel are in a state of rigid body rotation with uniform angular velocity about an axis perpendicular to the planes of the plates. Fluid within the channel is permeated by a uniform transverse magnetic field applied in a direction which is parallel to the axis of rotation.

\section{Formulation of the Problem and its Solution}

Consider flow of a viscous, incompressible, electrically conducting and heat generating/absorbing fluid within a parallel plate vertical channel (i.e. $z=0$ to $z=L$ ) in a uniform porous medium in the presence of a uniform transverse magnetic field $B_{0}$ applied in a direction which is parallel to $z$-axis about which both the fluid and channel are in a state of rigid body rotation with uniform angular velocity $\Omega$. Plate $z=0$ of the channel is kept at uniform temperature $T_{0}$ whereas plate $z=L$ of the channel is maintained at an oscillating temperature $T_{0}+\left(T_{w}-T_{0}\right) \cos \omega^{\prime} t^{\prime} \cdot \omega^{\prime}, T_{w}$ and $t^{\prime}$ are, respectively, frequency of oscillations, temperature of the plate $z=L$ in steady state (i.e. when $\omega^{\prime}=0$ ) and time. Flow within the channel is induced by a periodic pressure gradient $\partial p / \partial x=2 R \cos \omega^{\prime} t^{\prime}$ applied in $x$-direction, $R$ being a constant. Physical model of the problem is presented in figure 1.

The equations of motion for a viscous, incompressible, electrically conducting and heat generating/absorbing fluid in a rotating medium are

$$
\frac{\partial \vec{q}}{\partial t^{\prime}}+(\vec{q} \cdot \nabla) \vec{q}+2 \Omega \hat{k} \times \vec{q}=-\frac{1}{\rho} \nabla p^{\prime}+v \nabla^{2} \vec{q}-\frac{v}{K^{\prime}} \vec{q}+\frac{1}{\rho}(\vec{J} \times \vec{B})+g \beta^{\prime}\left(T^{\prime}-T_{0}\right) \hat{i}
$$


Ohm's law for a moving conductor is

$$
\begin{aligned}
& \nabla . \vec{q}=0, \\
& \nabla \times \vec{E}=-\frac{\partial \vec{B}}{\partial t^{\prime}}, \\
& \nabla \times \vec{B}=\mu_{e} \vec{J}, \\
& \nabla \cdot \vec{B}=0,
\end{aligned}
$$

$$
\vec{J}=\sigma(\vec{E}+\vec{q} \times \vec{B}),
$$

and energy equation for the problem is

$$
\frac{\partial T^{\prime}}{\partial t^{\prime}}+(\vec{q} \cdot \nabla) T^{\prime}=\frac{k}{\rho C_{p}} \nabla^{2} T^{\prime}-\frac{Q_{0}}{\rho C_{p}}\left(T^{\prime}-T_{0}\right),
$$

where $\vec{q}, \vec{B}, \vec{J}, \vec{E}, T^{\prime}, \rho, v, K^{\prime}, g, \beta^{\prime}, \sigma, \mu_{e}, k, C_{p}, \hat{i}, \hat{k}, t^{\prime}, p^{\prime}$ and $Q_{0}$ are, respectively, fluid velocity, magnetic field, current density, electric field, fluid temperature, fluid density, kinematic coefficient of viscosity, permeability of porous medium, acceleration due to gravity, volumetric coefficient of thermal expansion, electrical conductivity, magnetic permeability, thermal conductivity, specific heat at constant pressure, unit vector along $x$-axis, unit vector along $z$-axis, time, pressure including centrifugal force and dimensional heat generation/absorption coefficient. It may be noted that $Q_{0}<0$ for heat generation and $Q_{0}>0$ for heat absorption.

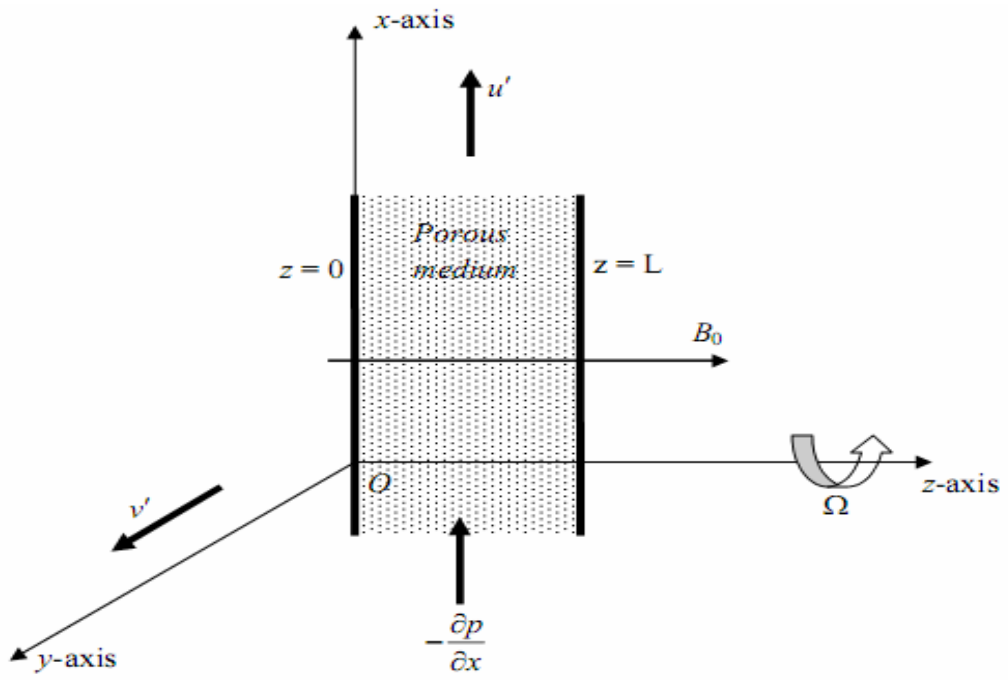

Figure 1. Physical model of the problem

Since plates of the channel are of infinite extent in $x$ and $y$ directions and electrically non-conducting and flow is fully developed so all physical quantities, except pressure $p^{\prime}$, depend on $z$ and $t^{\prime}$ only i.e pressure $p^{\prime}$ is function of $x, y, z$ and $t^{\prime}$ whereas fluid velocity $\vec{q}$, fluid temperature $T^{\prime}$, skin friction and Nusselt number are functions of $z$ and $t^{\prime}$ only.

Taking into consideration assumptions made above fluid velocity $\vec{q}$, magnetic field $\vec{B}$, current density $\vec{J}$ and electric field $\vec{E}$ are given by

$$
\vec{q} \equiv\left(u^{\prime}, v^{\prime}, 0\right), \vec{B} \equiv\left(B_{x}, B_{y}, B_{0}\right), \vec{J} \equiv\left(J_{x}, J_{y}, 0\right), \vec{E} \equiv\left(E_{x}, E_{y}, E_{z}\right),
$$

which are in agreement with the fundamental equations of Magnetohydrodynamics i.e. equations (1) to (6).

It is assumed that the induced magnetic field produced by motion of fluid is negligible in comparison to the applied one so that $\vec{B} \equiv\left(0,0, B_{0}\right)$. This assumption is valid because magnetic Reynolds number is very small for metallic liquids and partially ionized fluids (Cramer and Pai, 1973). Also no external electric field is applied so the effect of polarization of fluid is neglected (Meyer, 1958) i.e $\vec{E} \equiv(0,0,0)$.

Under the above assumptions, equation (1) with the help of (6) and equation (7) reduce to 


$$
\begin{gathered}
\frac{\partial u^{\prime}}{\partial t^{\prime}}-2 \Omega v^{\prime}=-\frac{1}{\rho} \frac{\partial p^{\prime}}{\partial x}+v \frac{\partial^{2} u^{\prime}}{\partial z^{2}}-\frac{v}{K^{\prime}} u^{\prime}-\frac{\sigma B_{0}^{2}}{\rho} u^{\prime}+g \beta^{\prime}\left(T^{\prime}-T_{0}\right) \\
\frac{\partial v^{\prime}}{\partial t^{\prime}}+2 \Omega u^{\prime}=v \frac{\partial^{2} v^{\prime}}{\partial z^{2}}-\frac{v}{K^{\prime}} v^{\prime}-\frac{\sigma B_{0}{ }^{2}}{\rho} v^{\prime} \\
0=-\frac{1}{\rho} \frac{\partial p^{\prime}}{\partial z} \\
\frac{\partial T^{\prime}}{\partial t^{\prime}}=\frac{k}{\rho c_{p}} \frac{\partial^{2} T^{\prime}}{\partial z^{2}}-\frac{Q_{0}}{\rho c_{p}}\left(T^{\prime}-T_{0}\right)
\end{gathered}
$$

We have considered oscillatory Hartmann convective flow so pressure $p^{\prime}$ is assumed in the following form

$$
p^{\prime}=2 R x \cos \left(\omega^{\prime} t^{\prime}\right)+F(y)+G(z) .
$$

It is noticed from equations (9), (10), (11) and (13) that pressure $p^{\prime}$ is constant along the axis of rotation i.e. $\frac{\partial p^{\prime}}{\partial z}=G^{\prime}(z)=0$. The absence of pressure gradient term $\frac{\partial p^{\prime}}{\partial y}=F^{\prime}(y)$ in equation (10) implies that there is a net cross flow in $y$-direction (Prasad Rao et $a l$, 1982). Buoyancy term $g \beta^{\prime}\left(T-T_{0}\right)$ is considered in equation (1) only because free-convection in this problem takes place under gravitational force (Singh, 1983; Tokis, 1986, 1988; Kythe and Puri, 1988 a, b; Nanousis, 1992 and Jha and Ajibade, 2009). Boundary conditions for the fluid velocity are hydrodynamic slip boundary conditions which are given by

$$
\left.\begin{array}{c}
\mu \frac{\partial u^{\prime}}{\partial z}=-\beta u^{\prime} \text { and } \mu \frac{\partial v^{\prime}}{\partial z}=-\beta v^{\prime} \quad \text { at } z=0, \\
\mu \frac{\partial u^{\prime}}{\partial z}=\beta u^{\prime} \text { and } \mu \frac{\partial v^{\prime}}{\partial z}=\beta v^{\prime} \quad \text { at } z=L .
\end{array}\right\}
$$

Boundary conditions (14) for the fluid velocity are well known hydrodynamic slip boundary conditions derived by Beavers and Joseph (1967). Here $\mu$ and $\beta$ are, respectively, coefficient of dynamic viscosity and coefficient of sliding friction.

Boundary conditions for the fluid temperature are

$$
\left.\begin{array}{l}
T^{\prime}=T_{0} \text { at } z=0 \\
T^{\prime}=T_{0}+\left(T_{w}-T_{0}\right) \cos \omega^{\prime} t^{\prime} \text { at } z=L,
\end{array}\right\}
$$

where $T_{0}<T^{\prime}<T_{w}$.

Equations (9), (10) and (12), in non-dimensional form, become

$$
\begin{gathered}
\frac{\partial u}{\partial t}-2 K^{2} v=-\frac{\partial p}{\partial \zeta}+\frac{\partial^{2} u}{\partial \eta^{2}}-\frac{1}{K_{1}} u-M^{2} u+G_{r} T, \\
\frac{\partial v}{\partial t}+2 K^{2} u=\frac{\partial^{2} v}{\partial \eta^{2}}-\frac{1}{K_{1}} v-M^{2} v, \\
\frac{\partial T}{\partial t}=\frac{1}{P_{r}} \frac{\partial^{2} T}{\partial \eta^{2}}-\phi T
\end{gathered}
$$

where

$\zeta=x / L, \eta=z / L, u=u^{\prime} L / v, v=v^{\prime} L / v, t=t^{\prime} v / L^{2}, p=L^{2} p^{\prime} / \rho v^{2}, T=\left(T^{\prime}-T_{0}\right) /\left(T_{w}-T_{0}\right)$, $K^{2}=\Omega L^{2} / v, K_{1}=K^{\prime} / L^{2}, M^{2}=B_{0}^{2} L^{2}(\sigma / \rho v), P_{r}=v \rho c_{p} / k, G_{r}=g \beta^{\prime}\left(T_{w}-T_{0}\right) L^{3} / v^{2}$ and $\phi=Q_{0} L^{2} / v \rho c_{p}$.

$K^{2}, M^{2}, G_{r}, P_{r}, K_{1}$ and $\phi$ are rotation parameter which is reciprocal of Ekman number, magnetic parameter which is square of Hartmann number, Grashof number, Prandtl number, permeability parameter and heat generation/absorption coefficient respectively.

Boundary conditions (14) and (15), in dimensionless form, are

$$
\begin{gathered}
\begin{array}{l}
u=-\alpha \frac{\partial u}{\partial \eta} \text { and } v=-\alpha \frac{\partial v}{\partial \eta} \text { at } \eta=0, \\
u=\alpha \frac{\partial u}{\partial \eta} \text { and } v=\alpha \frac{\partial v}{\partial \eta} \text { at } \eta=1,
\end{array} \\
T=0 \text { at } \eta=0 \text { and } T=\cos \omega t \text { at } \eta=1,
\end{gathered}
$$


where $\alpha=\mu / \beta L$ is slip parameter and $\omega=\omega^{\prime} L^{2} / v$ is frequency parameter.

Equations (16) and (17), in compact form, become

$$
\frac{\partial F}{\partial t}+2 i K^{2} F=-\frac{\partial p}{\partial \zeta}+\frac{\partial^{2} F}{\partial \eta^{2}}-\frac{1}{K_{1}} F-M^{2} F+G_{r} T
$$

where $F=u+i v$.

Boundary conditions (19), in compact form, are

$$
F+\alpha \frac{\partial F}{\partial \eta}=0 \text { at } \eta=0 \text { and } F-\alpha \frac{\partial F}{\partial \eta}=0 \text { at } \eta=1 .
$$

It may be noted that the fluid flow past a plate may be induced due to either by motion of the plate or free stream or by heating of the fluid or by both (Singh, 1983; Tokis, 1986, 1988; Kythe and Puri, 1988 a, b; Kim, 2000; Chamkha, 2004 and Mbeledogu and Ogulu, 2007). Convective fluid flow within the channel may be induced due to either by heating of the fluid (Jha and Ajibade, 2009) or by the movement of one of the plates of the channel and heating of the fluid (Singh and Kumar, 2009) or by applied pressure gradient and heating of the fluid (Prasad Rao et al, 1982; Ghosh and Bhattacharjee, 2000; Seth and Singh, 2008 and Seth and Ansari, 2009). We have considered oscillatory Hartmann convective flow so fluid flow, in our case, is induced due to applied oscillatory pressure gradient and by heating of the fluid because of temperature difference between lower and upper plates.

Therefore, pressure gradient $\frac{\partial p}{\partial \varsigma}$, fluid velocity $F(\eta, t)$ and fluid temperature $T(\eta, t)$ are assumed, in non-dimensional form, as

$$
\begin{gathered}
\frac{\partial p}{\partial \varsigma}=R\left(e^{i \omega t}+e^{-i \omega t}\right), \\
F(\eta, t)=F_{1}(\eta) e^{i \omega t}+F_{2}(\eta) e^{-i \omega t}, \\
T(\eta, t)=T_{1}(\eta) e^{i \omega t}+T_{2}(\eta) e^{-i \omega t},
\end{gathered}
$$

where $R<0$ for favourable pressure.

Equations (18) and (21) with the use of (23) and (24) reduce to

$$
\begin{gathered}
\frac{d^{2} T_{1}}{d \eta^{2}}-P_{r}(\phi+i \omega) T_{1}=0, \\
\frac{d^{2} T_{2}}{d \eta^{2}}-P_{r}(\phi-i \omega) T_{2}=0, \\
\frac{d^{2} F_{1}}{d \eta^{2}}-\left\{\frac{1}{K_{1}}+M^{2}+i\left(2 K^{2}+\omega\right)\right\} F_{1}=R-G_{r} T_{1}, \\
\frac{d^{2} F_{2}}{d \eta^{2}}-\left\{\frac{1}{K_{1}}+M^{2}+i\left(2 K^{2}-\omega\right)\right\} F_{2}=R-G_{r} T_{2} .
\end{gathered}
$$

Boundary conditions (20) and (22) become

$$
\left.\begin{array}{c}
T_{1}=0 \text { and } T_{2}=0 \text { at } \eta=0, \\
T_{1}=1 / 2 \text { and } T_{2}=1 / 2 \text { at } \eta=1,
\end{array}\right\}
$$

Equations (25) to (28) subject to boundary conditions (29) and (30) are solved and the solution for fluid temperature and fluid velocity is presented in the following form

$$
\begin{gathered}
T(\eta, t)=\frac{1}{2}\left[\frac{\sinh m_{1} \eta}{\sinh m_{1}} e^{i \omega t}+\frac{\sinh m_{3} \eta}{\sinh m_{3}} e^{-i \omega t}\right], \\
F(\eta, t)=\left\{C_{1} \cosh m_{2} \eta+C_{2} \sinh m_{2} \eta-\frac{R}{m_{2}{ }^{2}}-\frac{G_{r} \sinh m_{1} \eta}{2\left(m_{1}{ }^{2}-m_{2}{ }^{2}\right) \sinh m_{1}}\right\} e^{i \omega t}+
\end{gathered}
$$




$$
+\left\{C_{3} \cosh m_{4} \eta+C_{4} \sinh m_{4} \eta-\frac{R}{m_{4}{ }^{2}}-\frac{G_{r} \sinh m_{3} \eta}{2\left(m_{3}{ }^{2}-m_{4}{ }^{2}\right) \sinh m_{3}}\right\} e^{-i \omega t},
$$

where

$$
\begin{aligned}
& m_{1}=\left[P_{r}(\phi+i \omega)\right]^{1 / 2}, m_{2}=\left[\frac{1}{K_{1}}+M^{2}+i\left(2 K^{2}+\omega\right)\right]^{1 / 2}, \\
& m_{3}=\left[P_{r}(\phi-i \omega)\right]^{1 / 2}, m_{4}=\left[\frac{1}{K_{1}}+M^{2}+i\left(2 K^{2}-\omega\right)\right]^{1 / 2}, \\
& m_{5}=\left[\left(1+\alpha^{2} m_{2}^{2}\right) \sinh m_{2}-2 \alpha m_{2} \cosh m_{2}\right]^{-1}, \\
& m_{6}=\left[\left(1+\alpha^{2} m_{4}^{2}\right) \sinh m_{4}-2 \alpha m_{4} \cosh m_{4}\right]^{-1}, \\
& C_{1}=-m_{5}\left[\frac{R}{m_{2}}\left\{\alpha\left(1+\cosh m_{2}\right)-\frac{1}{m_{2}} \sinh m_{2}\right\}+\frac{\alpha m_{2} G_{r}}{2\left(m_{1}^{2}-m_{2}^{2}\right)}\left\{1-\frac{\alpha m_{1}}{\sinh m_{1}}\left(\frac{\sinh m_{2}}{\alpha m_{2}}+\cosh m_{1}-\cosh m_{2}\right)\right\}\right], \\
& C_{2}=m_{5}\left[\frac{G_{r}}{2\left(m_{1}^{2}-m_{2}^{2}\right)}\left\{1-\frac{\alpha m_{1}}{\sinh m_{1}}\left(\cosh m_{1}+\cosh m_{2}-\alpha m_{2} \sinh m_{2}\right)\right\}+\frac{R}{m_{2}^{2}}\left(1-\cosh m_{2}+\alpha m_{2} \sinh m_{2}\right)\right], \\
& C_{3}=-m_{6}\left[\frac{R}{m_{4}}\left\{\alpha\left(1+\cosh m_{4}\right)-\frac{1}{m_{4}} \sinh m_{4}\right\}+\frac{\alpha m_{4} G_{r}}{2\left(m_{3}^{2}-m_{4}^{2}\right)}\left\{1-\frac{\alpha m_{3}}{\sinh m_{3}}\left(\frac{\sinh m_{4}}{\alpha m_{4}}+\cosh m_{3}-\cosh m_{4}\right)\right\}\right], \\
& C_{4}=m_{6}\left[\frac{G_{r}}{2\left(m_{3}^{2}-m_{4}^{2}\right)}\left\{1-\frac{\alpha m_{3}}{\sinh m_{3}}\left(\cosh m_{3}+\cosh m_{4}-\alpha m_{4} \sinh m_{4}\right)\right\}+\frac{R}{m_{4}{ }^{2}}\left(1-\cosh m_{4}+\alpha m_{4} \sinh m_{4}\right)\right],
\end{aligned}
$$

\section{Asymptotic Solution}

In order to gain further insight into the flow pattern, asymptotic behavior of the solution (32) will be analyzed for large values of frequency parameter $\omega$.

i.e. when $\omega>>1, \mathrm{M}^{2} \sim O(1)$ and $K^{2} \sim O(1)$

When $\omega$ is large, boundary layer type flow is expected. For the boundary layer flow near the plate $\eta=1$, introducing boundary layer coordinate $\xi=1-\eta$, the asymptotic solution for the fluid velocity is obtained from (32) and is presented in the following form

$$
\begin{aligned}
& u=\lambda\left[-\sqrt{P_{r}}\left\{e^{-\alpha_{1} \xi} \sin \left(\omega t-\beta_{1} \xi\right)+e^{-\alpha_{2} \xi} \sin \left(\omega t-\beta_{2} \xi\right)\right\}+2 e^{-\alpha_{3} \xi} \sin \left(\omega t-\beta_{3} \xi\right)\right]+\frac{2 R}{\omega}(\cos \omega t+\pi / 2), \\
& v=\lambda \sqrt{P_{r}}\left[e^{-\alpha_{1} \xi} \cos \left(\omega t-\beta_{1} \xi\right)-e^{-\alpha_{2} \xi} \cos \left(\omega t-\beta_{2} \xi\right)\right],
\end{aligned}
$$

where

$$
\begin{aligned}
\lambda & =\frac{G_{r}}{2 \omega\left(1-P_{r}\right)}, \\
\alpha_{1} & =\sqrt{\frac{\omega}{2}}\left\{1+\frac{K^{2}}{\omega}+\frac{1+K_{1} M^{2}}{2 \omega K_{1}}\right\}, \beta_{1}=\sqrt{\frac{\omega}{2}}\left\{1+\frac{K^{2}}{\omega}-\frac{1+K_{1} M^{2}}{2 \omega K_{1}}\right\}, \\
\alpha_{2} & =\sqrt{\frac{\omega}{2}}\left\{1-\frac{K^{2}}{\omega}+\frac{1+K_{1} M^{2}}{2 \omega K_{1}}\right\}, \beta_{2}=\sqrt{\frac{\omega}{2}}\left\{1-\frac{K^{2}}{\omega}-\frac{1+K_{1} M^{2}}{2 \omega K_{1}}\right\}, \\
\alpha_{3} & =\sqrt{\frac{\omega P_{r}}{2}}\left(1+\frac{\phi}{2 \omega}\right), \beta_{3}=\sqrt{\frac{\omega P_{r}}{2}}\left(1-\frac{\phi}{2 \omega}\right) .
\end{aligned}
$$

The expressions (33) to (38) demonstrate the existence of triple boundary layers of thicknesses $O\left(\alpha_{1}^{-1}\right)$, $O\left(\alpha_{2}^{-1}\right)$ and $O\left(\alpha_{3}^{-1}\right)$ near the plate $\eta=1$. Two of the boundary layers of thicknesses $O\left(\alpha_{1}^{-1}\right)$ and $O\left(\alpha_{2}^{-1}\right)$ may be identified as modified 
Sokes-Ekman boundary layers and can be viewed as classical Stokes-Ekman boundary layers modified by magnetic field and porosity of medium. The third boundary layer of thickness $O\left(\alpha_{3}^{-1}\right)$ may be recognized as modified Stokes boundary layer and can be viewed as classical Stokes boundary layer modified by source/sink effect. Similar types of boundary layers are formed near the plate $\eta=0$ of the channel. Exponential terms in the expressions (33) and (34) damp out quickly as $\xi$ increases. When $\xi>\alpha_{2}^{-1}$ i.e. outside the boundary layer region, Eqs. (33) and (34) reduce to

$$
u \approx \frac{2 R}{\omega} \cos (\omega t+\pi / 2), v \approx 0 .
$$

It is evident from (39) that, in the central core region, fluid flows in the primary flow direction only and oscillates with the same frequency $\omega$ as applied pressure gradient but has a phase lead of $\pi / 2$ over it.

\section{Skin Friction and Nusselt Number}

The expressions for the skin friction $\tau$ and Nusselt number $N u$, which are measure of shear stress due to primary and secondary flows and rate of heat transfer at the plate $\eta=1$ respectively, are presented in the following form.

$$
\begin{aligned}
\tau=\left(\tau_{x}+i \tau_{y}\right) & =\left\{C_{1} m_{2} \sinh m_{2}+C_{2} m_{2} \cosh m_{2}-\frac{G_{r} m_{1}}{2\left(m_{1}{ }^{2}-m_{2}{ }^{2}\right)} \operatorname{coth} m_{1}\right\} e^{i \omega t}+ \\
& +\left\{C_{3} m_{4} \sinh m_{4}+C_{4} m_{4} \cosh m_{4}-\frac{G_{r} m_{3}}{2\left(m_{3}{ }^{2}-m_{4}{ }^{2}\right)} \operatorname{coth} m_{3}\right\} e^{-i \omega t},
\end{aligned}
$$

and

$$
N u=-\frac{1}{2}\left[m_{1} \operatorname{coth} m_{1} e^{i \omega t}+m_{3} \operatorname{coth} m_{3} e^{-i \omega t}\right] .
$$

\section{Results and Discussion}

To study the effects of wall slip, magnetic field, rotation, thermal buoyancy force, porosity of medium, oscillations and thermal source/sink on the flow-field numerical values of both primary and secondary fluid velocities, computed from analytical solution reported in Section 2 by MATLAB software, are displayed graphically versus channel width variable $\eta$ for various values of slip parameter $\alpha$, magnetic parameter $M^{2}$, rotation parameter $K^{2}$, Grashof number $G_{r}$, permeability parameter $K_{1}$, frequency parameter $\omega$, heat generation coefficient $\phi(<0)$ and heat absorption coefficient $\phi(>0)$ in Figs. 2 to 15 taking $P_{r}=0.71$, $\omega t=\pi / 2$ and $R=-1$. It is evident from Figs. 2 to 5 that primary velocity $u$ and secondary velocity $v$ decrease on increasing either slip parameter $\alpha$ or magnetic parameter $M^{2}$ for both heat generating and absorbing fluids which implies that wall slip and magnetic field have tendency to retard fluid flow in the primary and secondary flow directions for both heat generating and absorbing fluids. Figures 6 and 7 show that, for both heat generating and absorbing fluids, primary velocity $u$ decreases whereas secondary velocity $v$ increases with the increase in rotation parameter $K^{2}$ which implies that, for both heat generating and absorbing fluids, rotation tends to retard fluid flow in the primary flow direction whereas it has reverse effect on the fluid flow in secondary flow direction. Figures 8 to 13 reveal that, for both heat generating and absorbing fluids, $u$ and $v$ increase on increasing either $G_{r}$ or $K_{1}$ or $\omega$ which implies that, for both heat generating and absorbing fluids, thermal buoyancy force, porosity of medium and oscillations have tendency to accelerate fluid flow in both the primary and secondary flow directions. It is noticed from Figs. 14 and 15 that $u$ and $v$ increase on increasing $\phi(<0)$ and decrease on increasing $\phi(>0)$ which implies that thermal source accelerates fluid flow in both the primary and secondary flow directions whereas thermal sink has reverse effect on it. 


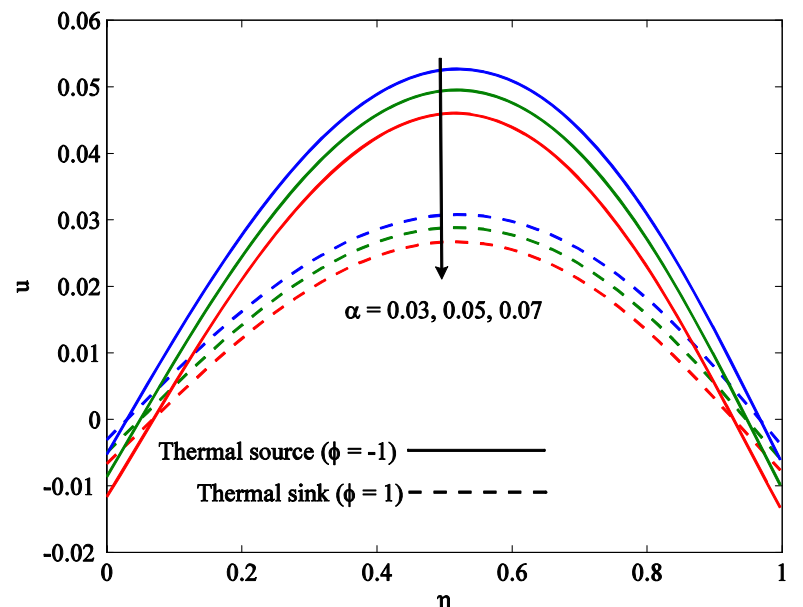

Figure 2. Profiles of primary velocity when $M^{2}=4, K^{2}=3, G_{r}=2, K_{1}=0.2$ and $\omega=3$.

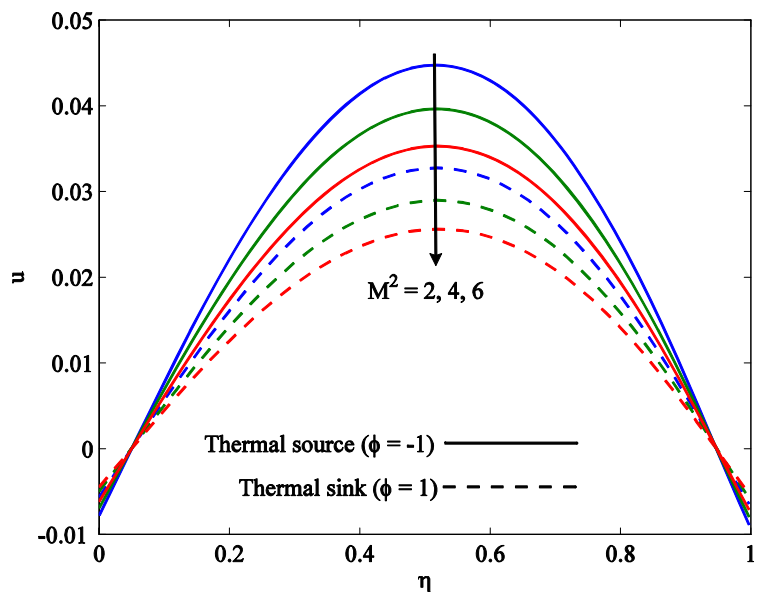

Figure 4. Profiles of primary velocity when $\alpha=0.05, \mathrm{~K}^{2}=3, \mathrm{G}_{\mathrm{r}}=2$, $\mathrm{K}_{1}=0.2$ and $\omega=3$.

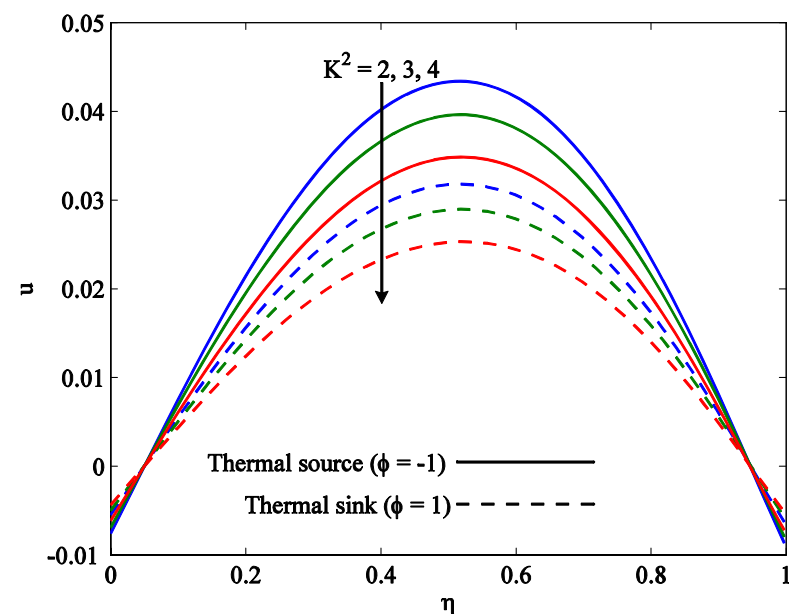

Figure 6. Profiles of primary velocity when $\alpha=0.05, \mathrm{M}^{2}=4, \mathrm{G}_{\mathrm{r}}=2$, $\mathrm{K}_{1}=0.2$ and $\omega=3$.

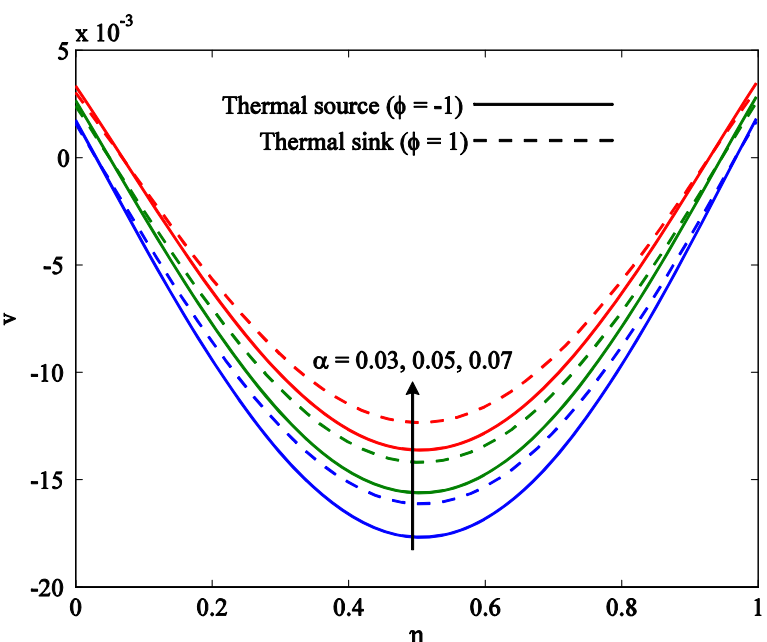

Figure 3. Profiles of secondary velocity when $M^{2}=4, K^{2}=3, G_{r}=2$, $\mathrm{K}_{1}=0.2$ and $\omega=3$.

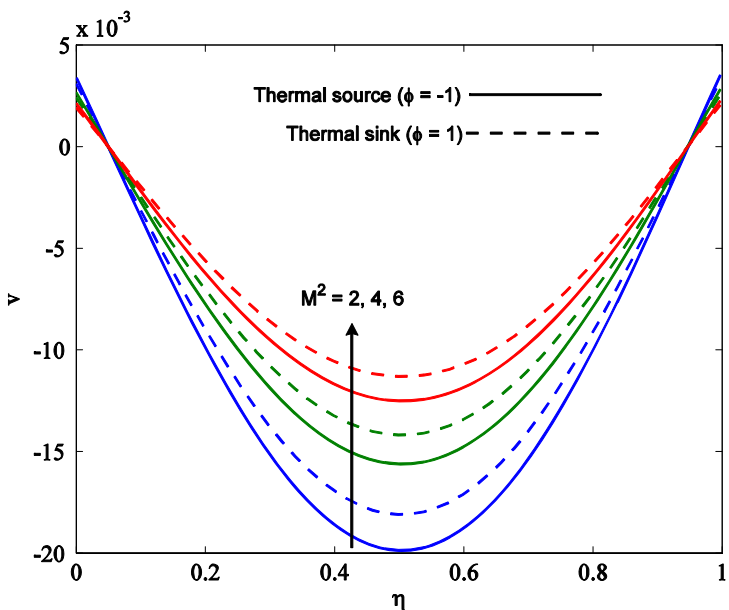

Figure 5. Profiles of secondary velocity when $\alpha=0.05, \mathrm{~K}^{2}=3, \mathrm{G}_{\mathrm{r}}=2$, $\mathrm{K}_{1}=0.2$ and $\omega=3$.

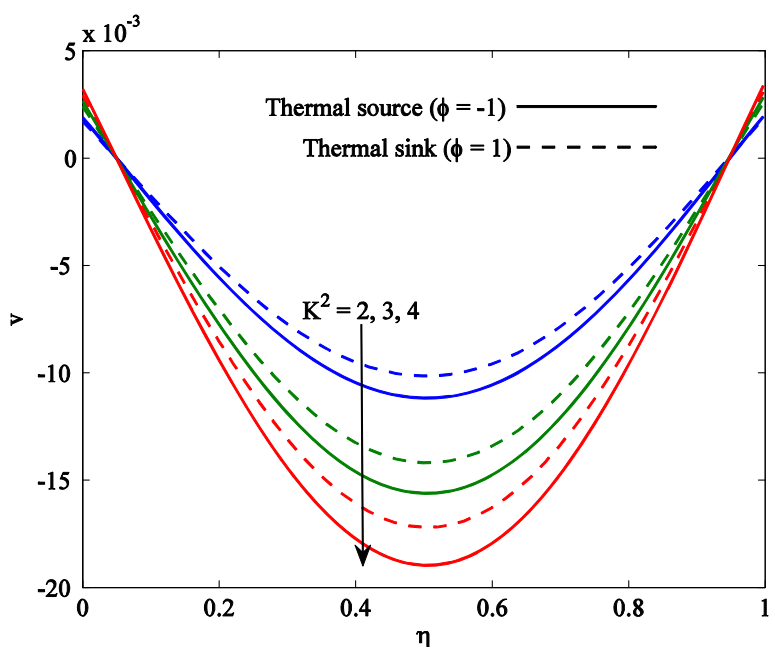

Figure 7. Profiles of secondary velocity when $\alpha=0.05, \mathrm{M}^{2}=4, \mathrm{G}_{\mathrm{r}}=2$, $\mathrm{K}_{1}=0.2$ and $\omega=3$. 


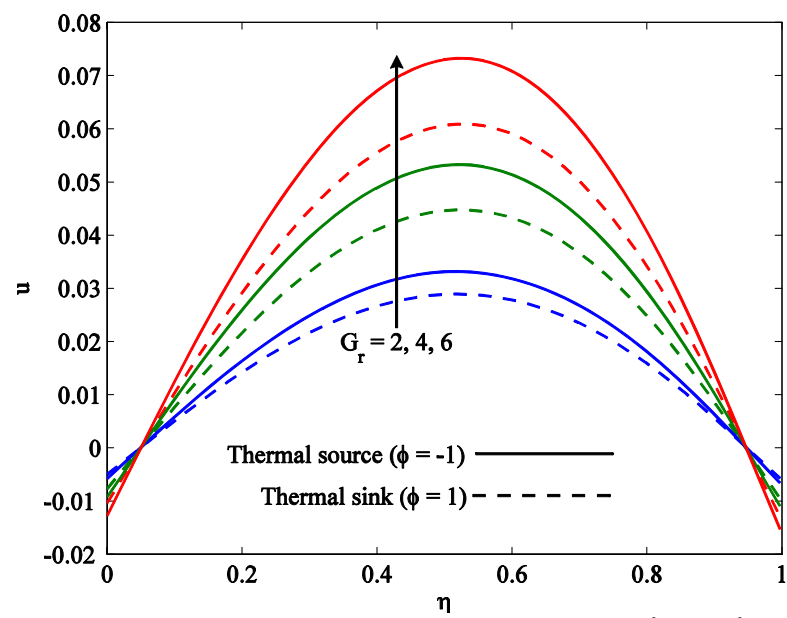

Figure 8. Profiles of primary velocity when $\alpha=0.05, \mathrm{M}^{2}=4, \mathrm{~K}^{2}=3$, $\mathrm{K}_{1}=0.2$ and $\omega=3$.

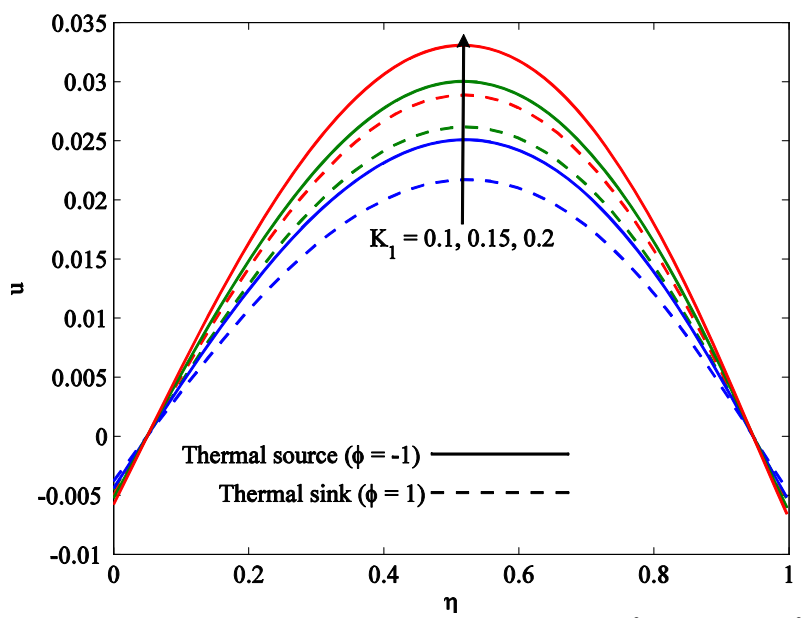

Figure 10. Profiles of primary velocity when $\alpha=0.05, \mathrm{M}^{2}=4, \mathrm{G}_{\mathrm{r}}=2, \mathrm{~K}^{2}=$ 3 and $\omega=3$.

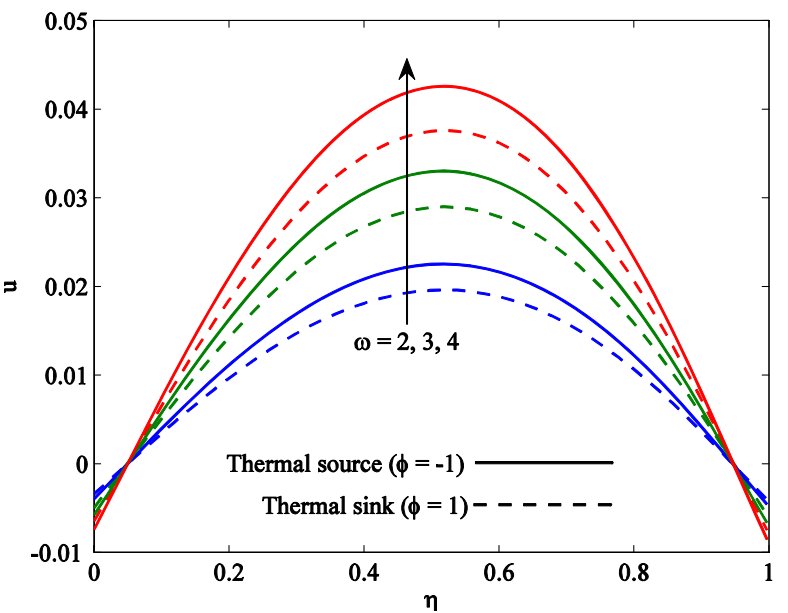

Figure 12. Profiles of primary velocity when $\alpha=0.05, \mathrm{M}^{2}=4, \mathrm{G}_{\mathrm{r}}=2, \mathrm{~K}^{2}=3$ and $K_{1}=0.2$.

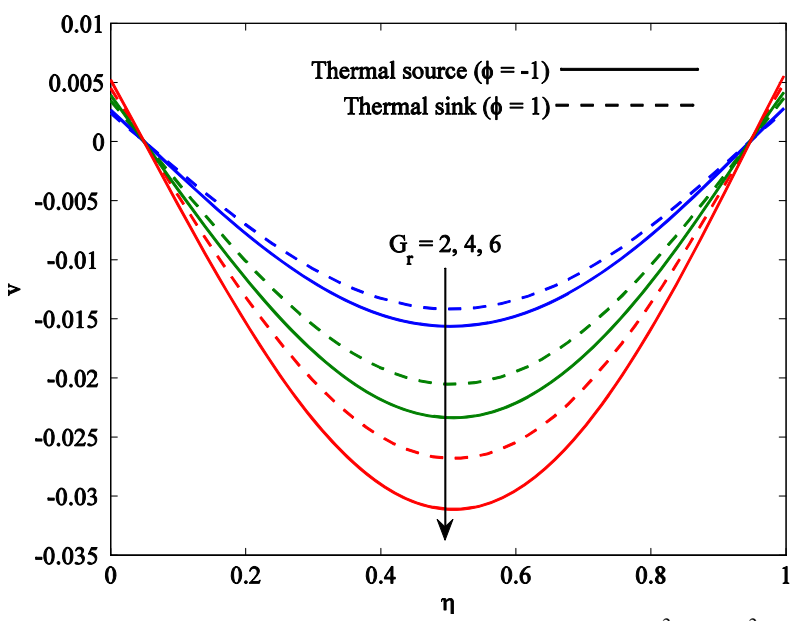

Figure 9. Profiles of secondary velocity when $\alpha=0.05, \mathrm{M}^{2}=4, \mathrm{~K}^{2}=3$, $\mathrm{K}_{1}=0.2$ and $\omega=3$.

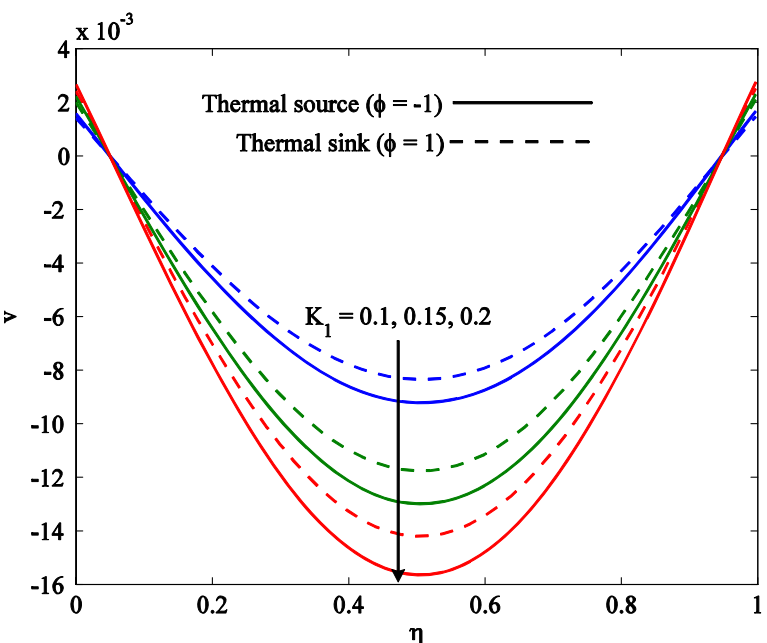

Figure 11. Profiles of secondary velocity when $\alpha=0.05, \mathrm{M}^{2}=4, \mathrm{G}_{\mathrm{r}}=2$, $\mathrm{K}^{2}=3$ and $\omega=3$

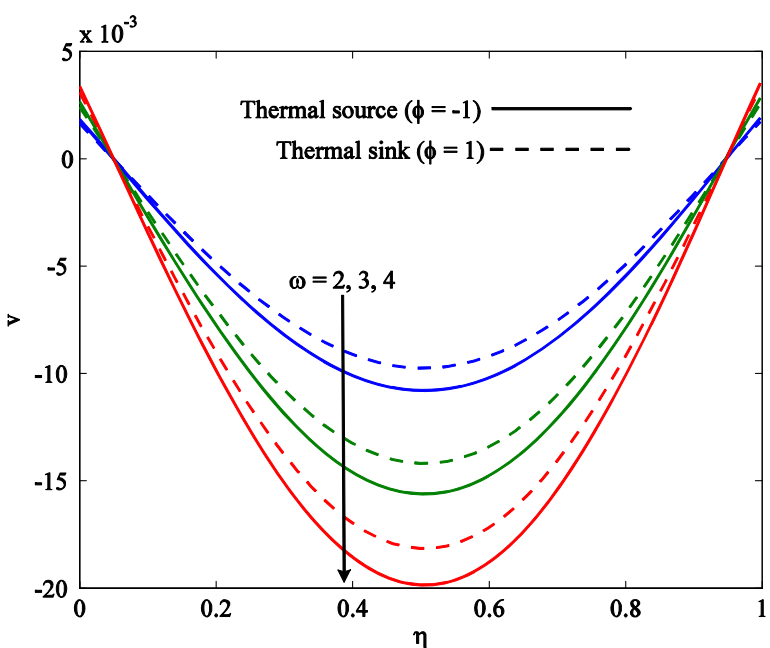

Figure 13. Profiles of secondary velocity when $\alpha=0.05, \mathrm{M}^{2}=4, \mathrm{G}_{\mathrm{r}}=2$, $\mathrm{K}^{2}=3$ and $K_{1}=0.2$. 


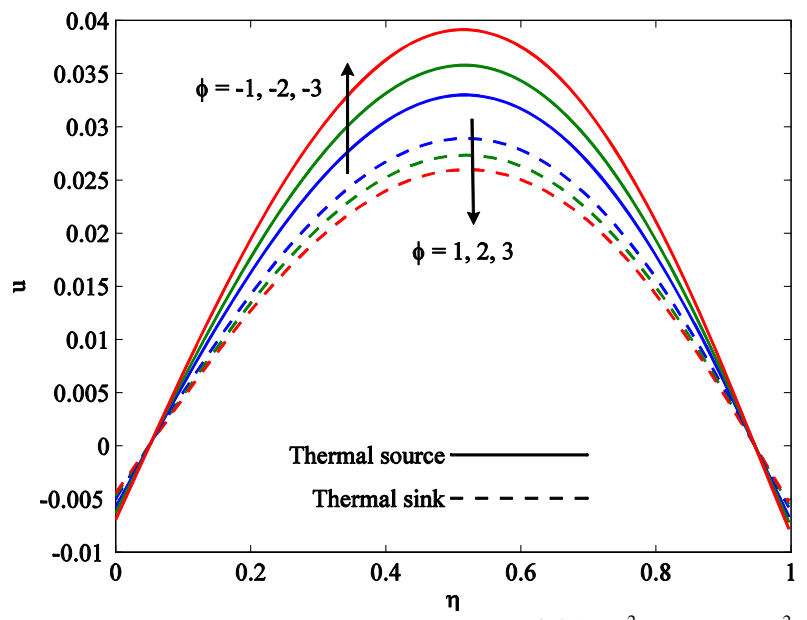

Figure 14. Profiles of primary velocity when $\alpha=0.05, \mathrm{M}^{2}=4, \mathrm{G}_{\mathrm{r}}=2, \mathrm{~K}^{2}=3$, $\omega=3$ and $K_{1}=0.2$.

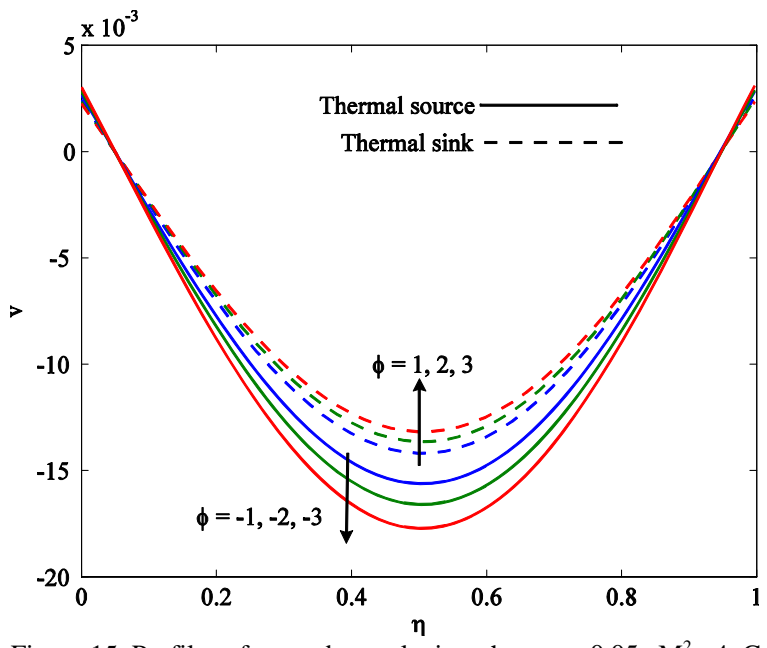

Figure 15. Profiles of secondary velocity when $\alpha=0.05, \mathrm{M}^{2}=4, \mathrm{G}_{\mathrm{r}}=2$, $\mathrm{K}^{2}=3, \omega=3$ and $K_{1}=0.2$.

The numerical values of fluid temperature, computed from analytical solution mentioned in Section 2 by MATLAB software, are depicted graphically in Figs. 16 to 18 for different values of heat generation coefficient $\phi(<0)$, heat absorption coefficient $\phi(>0)$, Prandtl number $P_{r}$ and frequency parameter $\omega$ taking $\omega t=\pi / 2$. Figure 16 reveals that fluid temperature $T$ increases on increasing $\phi(<0)$ and decreases on increasing $\phi(>0)$ which imply that thermal source tends to increase fluid temperature whereas thermal sink has reverse effect on it. Figure 17 shows that, for both heat generating and absorbing fluids, fluid temperature $T$ increases on increasing Prandtl number $P_{r}$. Since Prandtl number $P_{r}$ is ratio of viscosity to thermal diffusivity. An increase in thermal diffusivity leads to a decrease in Prandtl number. Therefore, thermal diffusion has tendency to reduce fluid temperature for both heat generating/absorbing fluids. It is noticed from Fig. 18 that, for both heat generating/absorbing fluids, fluid temperature $T$ decreases in the lower half of the channel whereas it decreases, attains a minimum and then increases in magnitude in the upper half of the channel on increasing $\omega$ which implies that there exists reverse flow of heat in the upper half of the channel due to oscillating temperature of plate $\eta=1$.

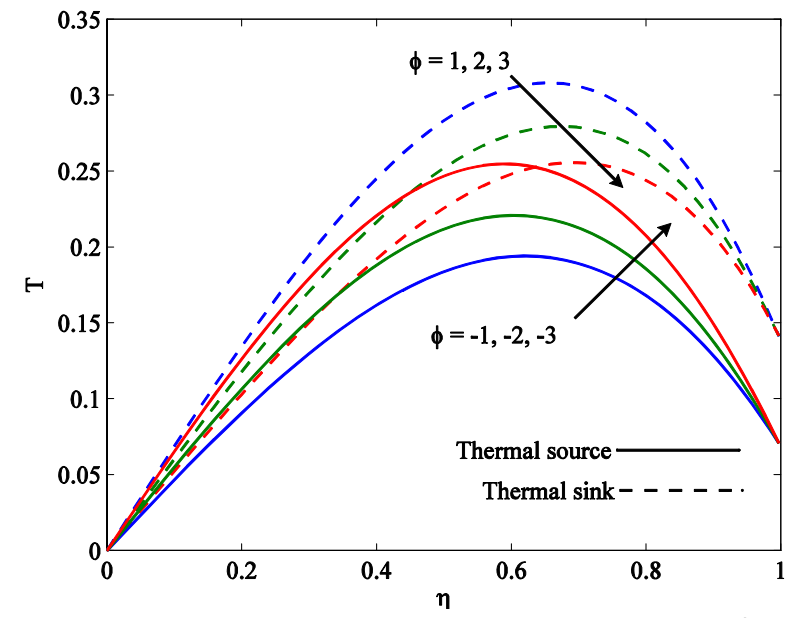

Figure 16. Temperature profiles when $\mathrm{P}_{\mathrm{r}}=0.71$ and $\omega=3$.

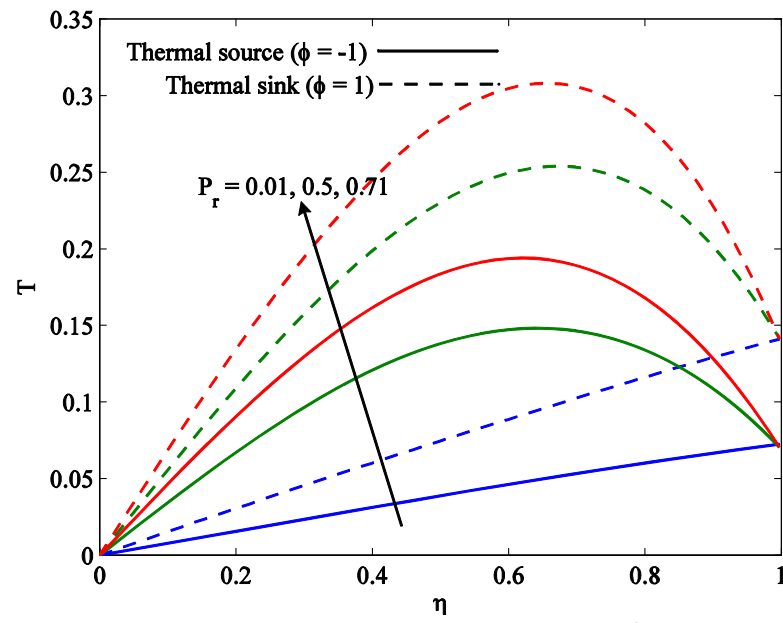

Figure 17. Temperature profiles when $\omega=3$. 


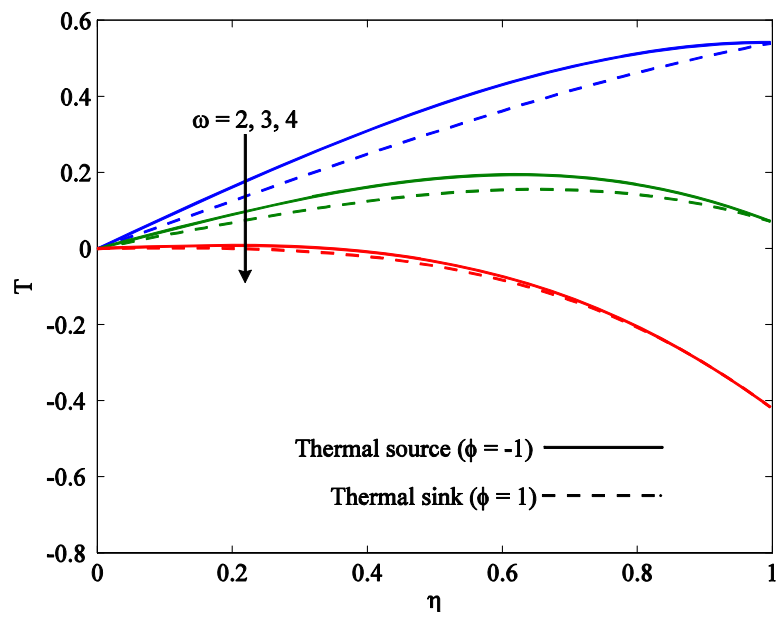

Figure 18. Temperature profiles when $\mathrm{P}_{\mathrm{r}}=0.71$.

The numerical values of primary and secondary skin frictions at plate $\eta=1$, computed from analytical expression reported in Section 4 by MATLAB software, are displayed in tabular form in Tables 1 to 8 for various values of $M^{2}, G_{r}, K^{2}, \phi, \omega, K_{1}$ and $\alpha$ taking $P_{r}=0.71, \omega t=\pi / 2$ and $R=-1$. It is evident from Tables 1 and 2 that, for both heat generating and absorbing fluids, primary skin friction $\tau_{x}$ and secondary skin friction $\tau_{y}$ decrease on increasing $M^{2}$ whereas these skin frictions increase on increasing $G_{r}$ which implies that, for both heat generating and absorbing fluids, magnetic field tends to reduce both primary and secondary skin frictions whereas thermal buoyancy force has reverse effect on it. It is noticed from Tables 3 and 4 that, for both heat generating and absorbing fluids, $\tau_{x}$ decreases whereas $\tau_{y}$ increases on increasing $K^{2}$ which implies that, for both heat generating and absorbing fluids, rotation tends to reduce primary skin friction and it has reverse effect on secondary skin friction. $\tau_{x}$ and $\tau_{y}$ increase on increasing $\phi(<0)$ and decrease on increasing $\phi(>0)$ which implies that thermal source has tendency to increase both primary and secondary skin frictions whereas thermal sink has reverse effect on it. It is found from Tables 5 and 6 that, for both heat generating and absorbing fluids, $\tau_{x}$ and $\tau_{y}$ increase on increasing either $\omega$ or $K_{1}$ which implies that oscillations and porosity of medium tend to increase primary and secondary skin frictions for both heat generating and absorbing fluids. It is revealed from Tables 7 and 8 that, for both heat generating and absorbing fluids, $\tau_{x}$ and $\tau_{y}$ decrease on increasing $\alpha$ which implies that, for both heat generating and absorbing fluids, wall slip tends to reduce primary and secondary skin friction.

Table 1. Skin frictions $\tau_{x}$ and $\tau_{y}$ when $\alpha=0.05, \mathrm{~K}^{2}=3, \omega=3, K_{1}=0.2$ and $\phi=-1$

\begin{tabular}{|c|c|c|c|c|c|c|}
\hline \multirow{2}{*}{$M^{2} \downarrow / G_{r} \rightarrow$} & \multicolumn{3}{|c|}{$-\tau_{x}$} & \multicolumn{3}{c|}{$\tau_{y}$} \\
\cline { 2 - 7 } & 2 & 4 & 6 & 2 & 4 & 6 \\
\hline 2 & 0.1473 & 0.2424 & 0.3374 & 0.0700 & 0.1046 & 0.1392 \\
\hline 4 & 0.1321 & 0.2191 & 0.3061 & 0.0553 & 0.0832 & 0.1111 \\
\hline 6 & 0.1192 & 0.1994 & 0.2795 & 0.0444 & 0.0674 & 0.0903 \\
\hline
\end{tabular}

Table 2. Skin frictions $\tau_{x}$ and $\tau_{y}$ when $\alpha=0.05, \mathrm{~K}^{2}=3, \omega=3, K_{1}=0.2$ and $\phi=1$

\begin{tabular}{|c|c|c|c|c|c|c|}
\hline \multirow{2}{*}{$M^{2} \downarrow / G_{r} \rightarrow$} & \multicolumn{3}{|c|}{$-\tau_{x}$} & \multicolumn{3}{c|}{$\tau_{y}$} \\
\cline { 2 - 7 } & 2 & 4 & 6 & 2 & 4 & 6 \\
\hline 2 & 0.1293 & 0.2064 & 0.2835 & 0.0639 & 0.0924 & 0.1209 \\
\hline 4 & 0.1156 & 0.1860 & 0.2565 & 0.0503 & 0.0733 & 0.0962 \\
\hline 6 & 0.1040 & 0.1688 & 0.2337 & 0.0403 & 0.0592 & 0.0780 \\
\hline
\end{tabular}


Table 3. Skin frictions $\tau_{x}$ and $\tau_{y}$ when $\alpha=0.05, \mathrm{M}^{2}=4, \mathrm{G}_{\mathrm{r}}=2, \mathrm{~K}_{1}=0.2$ and $\omega=3$.

\begin{tabular}{|c|c|c|c|c|c|c|}
\hline \multirow{2}{*}{$\phi \downarrow / K^{2} \rightarrow$} & \multicolumn{3}{|c|}{$-\tau_{x}$} & \multicolumn{3}{c|}{$\tau_{y}$} \\
\cline { 2 - 7 } & 2 & 3 & 4 & 2 & 3 & 4 \\
\hline-1 & 0.1432 & 0.1321 & 0.1186 & 0.0394 & 0.0553 & 0.0673 \\
\hline-2 & 0.1547 & 0.1431 & 0.1289 & 0.0417 & 0.0585 & 0.0713 \\
\hline-3 & 0.1690 & 0.1566 & 0.1416 & 0.0445 & 0.0624 & 0.0762 \\
\hline
\end{tabular}

Table 4. Skin frictions $\tau_{x}$ and $\tau_{y}$ when $\alpha=0.05, \mathrm{M}^{2}=4, \mathrm{G}_{\mathrm{r}}=2, \mathrm{~K}_{1}=0.2$ and $\omega=3$.

\begin{tabular}{|c|c|c|c|c|c|c|}
\hline \multirow{2}{*}{$\phi \downarrow / K^{2} \rightarrow$} & \multicolumn{3}{|c|}{$-\tau_{x}$} & \multicolumn{3}{c|}{$\tau_{y}$} \\
\cline { 2 - 7 } & 2 & 3 & 4 & 2 & 3 & 4 \\
\hline 1 & 0.1258 & 0.1156 & 0.1031 & 0.0360 & 0.0503 & 0.0611 \\
\hline 2 & 0.1192 & 0.1092 & 0.0972 & 0.0346 & 0.0484 & 0.0587 \\
\hline 3 & 0.1135 & 0.1039 & 0.0922 & 0.0334 & 0.0467 & 0.0567 \\
\hline
\end{tabular}

Table 5. Skin frictions $\tau_{x}$ and $\tau_{y}$ when $\alpha=0.05, \mathrm{M}^{2}=4, \mathrm{G}_{\mathrm{r}}=2, \mathrm{~K}^{2}=3$ and $\phi=-1$.

\begin{tabular}{|c|c|c|c|c|c|c|}
\hline \multirow{2}{*}{$\omega \downarrow / K_{1} \rightarrow$} & \multicolumn{3}{|c|}{$-\tau_{x}$} & \multicolumn{3}{c|}{$\tau_{y}$} \\
\cline { 2 - 7 } & 0.10 & 0.15 & 0.20 & 0.10 & 0.15 & 0.20 \\
\hline 2 & 0.0706 & 0.0827 & 0.0901 & 0.0227 & 0.0317 & 0.0381 \\
\hline 3 & 0.1035 & 0.1212 & 0.1321 & 0.0330 & 0.0460 & 0.0553 \\
\hline 4 & 0.1340 & 0.1567 & 0.1707 & 0.0423 & 0.0587 & 0.0703 \\
\hline
\end{tabular}

Table 6. Skin frictions $\tau_{x}$ and $\tau_{y}$ when $\alpha=0.05, \mathrm{M}^{2}=4, \mathrm{G}_{\mathrm{r}}=2, \mathrm{~K}^{2}=3$ and $\phi=1$.

\begin{tabular}{|c|c|c|c|c|c|c|}
\hline \multirow{2}{*}{$\omega \downarrow / K_{1} \rightarrow$} & \multicolumn{3}{|c|}{$-\tau_{x}$} & \multicolumn{3}{c|}{$\tau_{y}$} \\
\cline { 2 - 7 } & 0.10 & 0.15 & 0.20 & 0.10 & 0.15 & 0.20 \\
\hline 2 & 0.0609 & 0.0717 & 0.0784 & 0.0204 & 0.0286 & 0.0345 \\
\hline 3 & 0.0898 & 0.1058 & 0.1156 & 0.0298 & 0.0418 & 0.0503 \\
\hline 4 & 0.1170 & 0.1377 & 0.1505 & 0.0385 & 0.0537 & 0.0644 \\
\hline
\end{tabular}

Table 7. Skin frictions $\tau_{x}$ and $\tau_{y}$ when $\mathrm{K}_{1}=0.2, \mathrm{M}^{2}=4, \mathrm{G}_{\mathrm{r}}=2, \mathrm{~K}^{2}=3$ and $\phi=-1$.

\begin{tabular}{|c|c|c|c|c|c|c|}
\hline \multirow{2}{*}{$\omega \downarrow / \alpha \rightarrow$} & \multicolumn{3}{|c|}{$-\tau_{x}$} & \multicolumn{3}{c|}{$\tau_{y}$} \\
\cline { 2 - 7 } & 0.03 & 0.05 & 0.07 & 0.03 & 0.05 & 0.07 \\
\hline 2 & 0.0923 & 0.0901 & 0.0855 & 0.0419 & 0.0381 & 0.0341 \\
\hline 3 & 0.1354 & 0.1321 & 0.1252 & 0.0607 & 0.0553 & 0.0494 \\
\hline 4 & 0.1753 & 0.1707 & 0.1615 & 0.0772 & 0.0703 & 0.0630 \\
\hline
\end{tabular}

Table 8. Skin frictions $\tau_{x}$ and $\tau_{y}$ when $\mathrm{K}_{1}=0.2, \mathrm{M}^{2}=4, \mathrm{G}_{\mathrm{r}}=2, \mathrm{~K}^{2}=3$ and $\phi=1$.

\begin{tabular}{|c|c|c|c|c|c|c|}
\hline \multirow{2}{*}{$\omega \downarrow / \alpha \rightarrow$} & \multicolumn{3}{|c|}{$-\tau_{x}$} & \multicolumn{3}{c|}{$\tau_{y}$} \\
\cline { 2 - 7 } & 0.03 & 0.05 & 0.07 & 0.03 & 0.05 & 0.07 \\
\hline 2 & 0.0812 & 0.0784 & 0.0732 & 0.0381 & 0.0345 & 0.0308 \\
\hline 3 & 0.1197 & 0.1156 & 0.1078 & 0.0555 & 0.0503 & 0.0449 \\
\hline 4 & 0.1561 & 0.1505 & 0.1401 & 0.0710 & 0.0644 & 0.0576 \\
\hline
\end{tabular}

Numerical values of Nusselt number $N u$, computed from analytical expression mentioned in Section 4 by MATLAB software, are presented in tabular form in Tables 9 and 10 for different values of $P_{r}, \omega$ and $\phi$ taking $\omega t=\pi / 2$. It is found from Tables 9 and 10 that, for both heat generating and absorbing fluids, $N u$ increases on increasing either $P_{r}$ or $\omega$ which implies that thermal diffusion tends to reduce rate of heat transfer at plate $\eta=1$ whereas oscillations have reverse effect on it for both heat generating and absorbing fluids. Also $N u$ increases on increasing $\phi(<0)$ and decreases on increasing $\phi(>0)$ which implies that thermal source has tendency to enhance rate of heat transfer at plate $\eta=1$ whereas thermal sink has reverse effect on it. 
Table 9. Nusselt number Nu when $\omega=3$.

\begin{tabular}{|c|c|c|c|c|c|c|}
\hline$P_{r} \downarrow / \phi \rightarrow$ & 1 & 2 & 3 & -1 & -2 & -3 \\
\hline 0.01 & 0.0100 & 0.0100 & 0.0100 & 0.0100 & 0.0100 & 0.0100 \\
\hline 0.5 & 0.4631 & 0.4369 & 0.4138 & 0.5273 & 0.5670 & 0.6134 \\
\hline 0.71 & 0.6341 & 0.5868 & 0.5468 & 0.7587 & 0.8424 & 0.9469 \\
\hline
\end{tabular}

Table 10. Nusselt number Nu when $\mathrm{P}_{\mathrm{r}}=0.71$.

\begin{tabular}{|c|c|c|c|c|c|c|}
\hline$\omega \downarrow / \phi \rightarrow$ & 1 & 2 & 3 & -1 & -2 & -3 \\
\hline 2 & 0.4281 & 0.3955 & 0.3679 & 0.5152 & 0.5744 & 0.6491 \\
\hline 3 & 0.6341 & 0.5868 & 0.5468 & 0.7587 & 0.8424 & 0.9469 \\
\hline 4 & 0.8309 & 0.7711 & 0.7200 & 0.9868 & 1.0900 & 1.2169 \\
\hline
\end{tabular}

\section{Conclusion}

Unsteady hydromagnetic convective flow of a viscous incompressible electrically conducting heat generating/absorbing fluid within a parallel plate rotating channel in a porous medium under slip boundary conditions is investigated. The significant findings are summarized below:

a). For both heat generating and absorbing fluids:

(i). wall slip and magnetic field have tendency to retard fluid flow in both the primary and secondary flow directions.

(ii). rotation tends to retard fluid flow in primary flow direction whereas it has reverse effect on fluid flow in secondary flow direction.

(iii). buoyancy force, porosity of medium and oscillations have tendency to accelerate fluid flow in both the primary and secondary flow direction.

b). Thermal source tends to accelerate fluid flow in both the primary and secondary flow directions whereas thermal sink has reverse effect on it.

c). (i). Thermal source tends to enhance fluid temperature whereas thermal sink has reverse effect on it.

(ii).Thermal diffusion has tendency to reduce fluid temperature for both heat generating and absorbing fluids.

(iii).Oscillations tend to induce reverse flow of heat for both heat generating and absorbing fluids in the upper half of the channel due to oscillating temperature of upper plate.

d). For both heat generating and absorbing fluids:

(i). magnetic field tends to reduce both primary and secondary skin frictions whereas thermal buoyancy force has reverse effect on it.

(ii). rotation tends to reduce primary skin friction whereas it has reverse effect on secondary skin friction.

(iii). oscillations and porosity of medium tend to increase both primary and secondary skin frictions whereas wall slip has reverse effect on it.

e). Thermal source has tendency to increase both primary and secondary skin frictions whereas thermal skink has reverse effect on it.

f). Thermal source has tendency to enhance rate of heat transfer at plate $\eta=1$ whereas thermal sink has reverse effect on it. Thermal diffusion tends to reduce rate of heat transfer at plate $\eta=1$ whereas oscillations have reverse effect on it for both heat generating and absorbing fluids.

Acknowledgement: We are grateful to the referees for their valuable comments and suggestions which helped us to improve the quality of this research paper. We are also thankful to University Grant Commission, New Delhi, India for providing financial assistance to carry out this research work.

\section{References}

Abelman, S., Momoniat, E. and Hayat, T., 2009a, Steady MHD flow of a third grade fluid in a rotating frame and porous space, Nonlin. Anal.: RWA, Vol. 10, pp. 3322.

Abelman, S., Momoniat, E. and Hayat, T., 2009b, Couette flow of a third grade fluid with rotating frame and slip condition, Nonlin. Anal.: RWA, Vol. 10, pp. 3329.

Baker, I., Faw, R.E. and Kulacki, F.A., 1976, Post-accident heat removal part I: heat transfer within an internally heated nonboiling liquid layer, Nucl. Sci. Eng., Vol. 61, pp. 222-230.

Beavers, G.S. and Joseph, D.D., 1967, Boundary conditions at a naturally permeable wall, J. Fluid Mech., Vol. 30, pp. 197. 
Blake, T.D., 1990, Slip between a liquid and a solid: D.M. Tolsoi’s (1952) theory reconsidered, Colloids Surf., Vol. 47, pp. 135.

Chamkha, A.J., 2004, Unsteady MHD convective heat and mass transfer past a semi- infinite vertical permeable moving plate with heat absorption, Int. J. Engng. Sci., Vol. 42, pp.217.

Cramer, K.R. and Pai, S.I., 1973, Magnetofluiddynamics for engineers and Applied physicists, McGraw Hill Book Company, New York.

Crepeau, J.C. and Clarksean, R., 1997, Similarity solutions of natural convection with internal heat generation, ASME J. Heat Transfer, Vol. 119, pp. 183.

Darhuber, A.A. and Troian, S.M., 2005, Principles of microfluidic actuation by modulation of surface stresses, Ann. Rev. Fluid Mech., Vol. 37, pp. 425.

Das, B. K., Guria, M. and Jana, R. N., 2008, Unsteady Couette flow in a rotating system, Meccanica, Vol. 43, pp. 517.

Delichatsios, M.A., 1988, Air entrainment into buoyant jet flames and pool fires in: P.J. DiNenno et al., (Eds.), The SFPA Handbook of Fire Protection Engineering, NFPA Publications, Quincy, MA, pp. 306.

Ganapathy, R., 1994, A note on oscillatory Couette flow in a rotating system, ASME J. Appl. Mech., Vol. 61, pp. 208.

Ghosh, S.K., 1993, "Unsteady hydromagnetic flow in a rotating channel with oscillating pressure gradient” J. Phys. Soc. Japan, Vol. 62, pp. 3893.

Ghosh, S.K., Beg, O.A. and Narahari, M., 2009, Hall effects on MHD flow in a rotating system with heat transfer characteristics, Meccanica, Vol. 44, pp. 741.

Ghosh, S.K. and Bhattacharjee, P.K., 2000, Magnetohydrodynamic convective flow in a rotating channel, Arch. Mech., Vol. 52, pp. 303.

Ghosh, S.K. and Pop, I., 2003, Hall effects on unsteady hydromagnetic flow in a rotating system with oscillatory pressure gradient, Int. J. Appl. Mech. Eng., Vol. 8, pp. 43.

Greenspan, H.P. and Howard, L.N., 1963, On a time dependent motion of a rotating fluid, J. Fluid. Mech., Vol. 17, pp. 385.

Guria, M., Das, S., Jana, R.N. and Ghosh, S.K., 2009, Oscillatory Couette flow in the presence of an inclined magnetic field, Meccanica, Vol.44, pp. 555.

Hayat, T., Nadeem, S. and Asghar, S., 2004, Hydromagnetic Couette flow of an Oldroyd-B fluid in a rotating system, Int. $J$. Engng. Sci., Vol. 42, pp. 65.

Hayat, T. and Hutter, K., 2004, Rotating flow of a second-order fluid on a porous plate, Int. J. Nonlinear. Mech., Vol. 39, pp. 767.

Hayat, T., Nadeem, S., Asghar, S. and Siddiqui, A. M., 2001, Fluctuating flow of a third-grade fluid on a porous plate in a rotating medium” Int. J. Nonlinear. Mech., Vol. 36, pp. 901.

Holton, J. R., 1965, The influence of viscous boundary layers on transient motions in a stratified rotating fluid, Int. J. Atmos. Sci., Vol. 22, pp. 402.

Jana, R.N. and Datta, N., 1980, Hall effects on MHD Couette flow in a rotating system, Czech. J. Phys., Vol. 30, pp. 659.

Jana, R.N., Datta, N. and Mazumder, B.S., 1977, Magnetohydrodynamic Couette flow and heat transfer in a rotating system, $J$. Phys. Soc. Japan, Vol. 42, pp. 1034.

Jha, B.K. and Ajibade, A.O., 2009, Free convective flow of heat generating/ absorbing fluid between vertical porous plates with periodic heat input, Int. Comm. Heat Mass Transfer, Vol. 36, pp. 624.

Kamel, M.H., 2001, Unsteady MHD convection through porous medium with combined heat and mass transfer with heat source/ sink, Energy Conversion and Management, Vol. 42, pp. 393.

Khaled, A.-R.A. and Vafai, K., 2004, The effect of the slip condition on Stokes and Couette flows due to an oscillating wall: exact solutions, Int. J. Nonlinear Mech., Vol. 39, pp. 795.

Kim, Y. J., 2000, Unsteady MHD convective heat transfer past a semi-infinite vertical porous plate with variable suction, Int. $J$. Engng. Sci., Vol. 38, pp. 833.

Kythe, P. K. and Puri, P., 1988a, Unsteady MHD free-convection flows on a porous plate with time dependent heating in a rotating medium, Astrophysics Space Sci., Vol. 143, pp. 51.

Kythe, P. K. and Puri, P., 1988b, Unsteady MHD free-convection oscillatory flow on a porous plate in a rotating medium, Astrophysics Space Sci., Vol. 149, pp. 107.

Linga Raju, T., 2007, Magnetohydrodynamic slip-flow regime in a rotating channel, J. Ind. Acad. Math., Vol. 29, pp. 27.

Makinde, O.D. and Osalusi, E., 2006, MHD steady flow in a channel with slip at the permeable boundaries, Rom. J. Phys., Vol. 51, pp. 319.

Mandal, G. and Mandal, K.K., 1983, Effect of Hall current on MHD Couette flow between thick arbitrary conducting plate in a rotating system, J. Phys. Soc. Japan, Vol. 53, pp. 470.

Mandal, G. and Mandal, K.K. and Choudhary, G., 1982, On combined effects of Coriolis force and Hall current on steady MHD Couette flow and Heat transfer, J. Phys. Soc. Japan, Vol. 51, pp. 2010.

Marques, W., Kremer, G.M. Jr. and Shapiro, F.M., 2000, Couette flow with slip and jump boundary conditions, Continuum Mech. Thermodynam., Vol. 12, pp. 379.

Mazumder, B.S., 1991, An exact solution of oscillatory Couette flow in a rotating system, ASME J. Appl. Mech., Vol. 58, pp. 1104. 
Mazumder, B.S., 1977, Effect of wall conductances on hydromagnetic flow and heat transfer in a rotating channel, Acta Mech., Vol. 28, pp. 85.

Mbeledogu, I. U. and Ogulu, A., 2007, Heat and mass transfer of an unsteady MHD natural convection flow of a rotating fluid past a vertical porous flat plate in the presence of radiative heat transfer, Int. J. Heat Mass Transfer, Vol. 50, pp. 1902.

McKenzie, D.P., Roberts, J.M. and Weiss, N.O., 1974, Convection in the Earth's mantle: towards a numerical simulation, J. Fluid Mech., Vol. 62, pp. 465.

Melin, T., Diesinger, H., Deresmes, D. and Stievenard, D., 2004, Probing nanoscale dipole-dipole interactions by electric force microscopy” Phys. Rev. Lett., Vol. 92, pp. 166101.

Meyer, R.C., 1958, On reducing aerodynamic heat-transfer rates by magnetohydrodynamic techniques J. Aero. Sci., pp. 25, pp. 561.

Moalem, D., 1976, Steady state heat transfer with porous medium with temperature dependent heat generation, Int. J. Heat Mass Transfer, Vol. 19, pp. 529.

Mukherjee, S. and Debnath, L., 1977, On unsteady rotating boundary layer flows between two porous plates, ZAMM, Vol. 57, pp. 188.

Nagy, T. and Demendy, Z., 1993, Influence of wall properties on Hartmann flow and heat transfer in a rotating system, Acta Phys. Hung., Vol. 7, pp. 291.

Nagy, T. and Demendy, Z., 1995, Effects of Hall currents and Coriolis force on Hartmann flow under general wall conditions, Acta Mech., Vol. 113, pp. 77.

Nanda, R.S. and Mohanty, H.K., 1971, Hydromagnetic flow in a rotating channel, Appl. Sci. Res., Vol. 24 , pp. 65.

Nanousis, N., 1992, Thermal-diffusion effects on MHD free-convective and mass-transfer flow past a moving infinite vertical plate in a rotating fluid, Astrophysics Space Sci., Vol. 191, pp. 313.

Prasad Rao, D.R.V., Krishna, D.V. and Debnath, L., 1982, Combined effect of free and forced convection on MHD flow in a rotating porous channel, Int. J. Math. \& Math. Sci., Vol. 5, pp. 165.

Puri, P., 1974, Rotating flow of an elastico-viscous fluid on an oscillatory plate, ZAMM, Vol. 54, pp. 743.

Puri, P. and Kulshrestha, P.K., 1974, Rotating flow of non-Newtonian fluids, Appl. Anal., Vol. 4, pp. 131.

Rahman, M.M. and Sattar, M.A., 1999, MHD free convection and mass transfer flow with oscillating plate velocity and constant heat source in a rotating frame of reference, The Dhaka Univ. J. Sci., Vol. 47, pp. 63.

Raman Rao, V.V. and Linga Raju, T., 1990, Hall effect in the viscous incompressible flow through a rotating channel between two porous walls, Def. Sci. J., Vol. 40, pp. 243.

Sastry, V.U.K. and Bhadram, C.V.V., 1976, Magnetogasdyanamic flow past an infinite porous plate in slip flow regime, Appl. Sci. Res., Vol. 32, pp. 415.

Seth, G.S. and Ansari, Md. S., 2009, Magnetohydrodynamic convective flow in a rotating channel with Hall effects, Int. J. Theo. Appl. Mech., Vol. 4, pp. 205.

Seth, G.S. and Maiti, M.K., 1982, MHD Couette flow and heat transfer in a rotating system, Ind. J. Pure Appl. Math., Vol. 13, pp. 931.

Seth, G.S., Nandkeolyar, R., Mahto, N. and Singh, S.K., 2009, MHD Couette flow in a rotating system in the presence of an inclined magnetic field, Appl. Math. Sci., Vol. 3, pp. 2919.

Seth, G.S. and Singh, M.K., 2008, Combined free and forced convection MHD flow in a rotating channel with perfectly conducting walls, Ind. J. Theo. Phys., Vol. 56, pp. 203.

Seth, G.S. and Jana, R.N., 1980, Unsteady hydromagnetic flow in a rotating channel with oscillating pressure gradient, Acta Mech., Vol. 37, pp. 29.

Siegmann, W.L., 1971, The spin-down of rotating stratified fluids, J. Fluid. Mech., Vol. 47, pp. 689.

Singh, A. K., 1983, MHD free-convection flow in the Stokes problem for a vertical porous plate in a rotating system, Astrophysics Space Sci., Vol. 95, pp. 283.

Singh, K.D., 2000, An oscillatory hydromagnetic Couette flow in a rotating system, ZAMM, Vol. 80, pp. 429.

Singh, K. D. and Kumar, R., 2009, Combined effects of Hall current and rotation on free convection MHD flow in a porous channel, Ind. J. Pure Appl. Phys., Vol.47, pp. 617.

Smolentsev, S., 2009, MHD duct flows under hydrodynamic slip condition, Theo. Comput. Fluid Dyn., Vol. 23 , pp. 557.

Soltani, F. and Tilmazer, U., 1998, Slip velocity and slip layer thickness in flow of concentrated suspensions, J. Appl. Polym. Sci., Vol. 70, pp. 515.

Soundalgekar, V.M., 1970, On hydromagnetic fluctuating flow past an infinite porous plate in slip flow regime, Appl. Sci. Res., Vol. 23, pp. 301.

Sparrow, E.M. and Cess, R.D., 1961, The effect of a magnetic field on free convection heat transfer, Int. J. Heat Mass Transfer, Vol. 3, pp. 267.

Tokis, J. N., 1986, Unsteady Magnetohydrodynamic free-convection flows in a rotating fluid, Astrophysics Space Sci., Vol. 119, pp. 305.

Tokis, J. N., 1988, Free-convection and mass transfer effects on the Magnetohydrodynamic flows near a moving plate in a rotating medium,, Astrophysics Space Sci., Vol. 144, pp. 291. 
Vajravelu, K. and Nayfeh, J., 1992, Hydromagnetic convection at a cone and a wedges, Int. Comm. Heat Mass Transfer, Vol. 19, pp.701.

Vidyanidhi, V., 1969, Secondary flow of a conducting liquid in a rotating channel, J. Math. Phys. Sci., Vol. 3, pp. 193.

Walin, G., 1969, Some aspects of time dependent motion of a stratified rotating fluid, J. Fluid. Mech., Vol. 36, pp. 289.

Westphal, B.R., Keiser, D.D., Rigg, R.H. and Laug, D.V., 1994, Production of metal waste forms from spent nuclear fuel treatment, DOE Spent Nuclear Fuel Conference, Salt Lake City, UT, pp. 288.

\section{Biographical notes}

Dr. G.S. Seth is an Associate Professor in the Department of Applied Mathematics, Indian School of Mines, Dhanbad, India. He received his Ph.D. degree in Mathematics from Indian Institute of Technology, Kharagpur, India. He has more than twenty nine years of experience in teaching and research. His current area of research studies includes Fluid dynamics, Magnetohydrodynamics and heat transfer. He was visiting Assistant Professor at University of Aden, Republic of Yemen during the period September 01, 1991 to August 31, 1993. He has published more than forty four research papers in national/international journals of repute.

R. Nandkeolyar received M.Sc. degree in Mathematics from Vinoba Bhave University, Hazaribag, India in the year 2005 and M.Phil. degree in Applied Mathematics from Indian School of Mines, Dhanbad, India in the year 2007. Subsequently he joined the department of Applied Mathematics, Indian School of Mines, Dhanbad, India as a Junior Research Fellow to do research work leading to Ph.D. degree on the topic entitled "An Investigation of Some Problems of Magnetohydrodynamic Flow and Heat Transfer”. He has submitted his Ph.D. thesis to Indian School of Mines, Dhanbad, India. He has three and half years of research experience and has published thirteen research papers in journals of national/international repute. At present he is Lecturer in Mathematics at GITAM University, Hyderabad, India.

Dr. Md. S. Ansari received his Ph.D. degree in Applied Mathematics and M.Sc. degree in Mathematics and Computing from Indian School of Mines, Dhanbad, India. He has four years of research experience. He has published fifteen research papers in national/international journals of repute. He is Assistant Professor in Mathematics, School of Petroleum Technology, Pandit Deendayal Petroleum University, Gandhinagar, Gujarat, India.

Received July 2010

Accepted November 2010

Final acceptance in revised form November 2010 